\title{
EFFECT OF ACUTE WHOLE-BODY VIBRATION EXERCISE WITH BLOOD FLOW RESTRICTION ON VASCULAR ENDOTHELIAL GROWTH FACTOR RESPONSE
}

\author{
Wu Chih-Min ${ }^{1}$, Chen Wen-Chyuan ${ }^{2,3}$, and Cai Zong-Yan ${ }^{4}$ \\ ${ }^{1}$ Department of Leisure and Sports Management, Cheng Shiu University, Kaohsiung City, Taiwan \\ ${ }^{2}$ Center for General Education, Chang Gung University of Science and Technology, \\ Taoyuan, Taiwan \\ ${ }^{3}$ Department of Otolaryngology-Head and Neck Surgery, \\ Linkou Chang Gung Memorial Hospital, Taoyuan, Taiwan \\ ${ }^{4}$ Division of Physical and Health Education, Center for General Education, \\ National Sun Yat-sen University, Kaohsiung City, Taiwan
}

Original scientific paper

https://doi.org/10.26582/k.50.2.8

UDC: 612.171-043.3

\begin{abstract}
:
This study aimed to investigate the effects of the whole-body vibration (WBV) exercise with and without blood flow restriction (BFR) on heart rate (HR), oxygen saturation $\left(\mathrm{SpO}_{2}\right)$, and circulating vascular endothelial growth factor (VEGF) response. Ten physically inactive male adults participated in this study and completed WBV (frequency: $26 \mathrm{~Hz}$; amplitude: $4 \mathrm{~mm}$; 10 sets for $1 \mathrm{~min}$, with 1-2 min of rest between sets) and WBV + BFR sessions in a repeated measures crossover design, with a 1-week interval separating the sessions. In the WBV + BFR session, participants wore a BFR device inflated to $140 \mathrm{mmHg}$ on the proximal portion of the thigh muscle. Results indicated that WBV + BFR caused a greater HR response than WBV alone $(\mathrm{p}<.05)$. Neither WBV + BFR nor WBV sessions caused a significant change in $\mathrm{SpO}_{2}(\mathrm{p}>$.05). Only the WBV + BFR session caused a significant increase in the VEGF response $(p<.05)$, and WBV + BFR elicited a significantly higher VEGF response than did WBV after exercise $(p<.05)$. In conclusion, an acute bout of WBV + BFR exercise magnifies the HR response relative to WBV exercise and induces an increase in circulating VEGF values. These alterations seem unrelated to systematic $\mathrm{SpO}_{2}$.
\end{abstract}

Key words: oxygen saturation, heart rate, physically inactive male adults

\section{Introduction}

In recent decades, an emerging resistance training method known as occlusion training or blood flow restriction (BFR) resistance training has attracted a great deal of attention. BFR resistance training allows individuals to train their muscles at a desired intensity while keeping the load relatively low, which benefits those who are unable to tolerate resistance training at heavy loads (Loenneke \& Pujol, 2009; Pope, Willardson, \& Schoenfeld, 2013). Based on this rationale, BFR resistance training seems to be both an effective and safe alternative to heavy load resistance training, particularly in frail individuals (Takarada, Takazawa, \& Ishii, 2000a), rehabilitating populations (Loenneke, Young, Wilson, \& Andersen, 2013), and even in athletes who perform a planned BFR resist- ance training program (Scott, Loenneke, Slattery, \& Dascombe, 2016).

BFR resistance exercise is an approach involving a low-load resistance exercise combined with BFR, which typically involves wrapping a pressure cuff or wraps around the proximal portion of exercising muscles (Loenneke \& Pujol, 2009). This training method has largely been reported to have an additive effect on strength training adaptations (e.g., muscle hypertrophy and strength gain) when compared with low-load resistance exercises without BFR (Loenneke \& Pujol, 2009; Pope, et al., 2013). Furthermore, muscle hypertrophy achieved by the BFR resistance exercise is similar to that achieved by a high-load resistance training (Kim, et al., 2017; Takarada, et al., 2000b; Vechin, et al., 2015). 
In addition to achieving increases in muscle mass and strength, BFR resistance training has recently demonstrated the ability to improve muscular endurance (Kacin \& Strazer, 2011). These adaptive changes were characterized by facilitating oxygen delivery within muscles. Physiological parameters relevant to muscle endurance, such as heart rate (HR) elicited significantly greater responses in BFR combined with resistance exercise relative to non-BFR resistance exercise (Neto, et al., 2016; Takano, et al., 2005). Moreover, a significant increase in skeletal muscle capillarization was proposed to contribute to increases in muscular endurance (Evans, Vance, \& Brown, 2010). However, limited studies have provided data regarding the acute effects of BFR resistance exercise on the expression of biomarkers associated with angiogenesis as an indirect measure of capillarization. Among several factors known to promote angiogenesis, vascular endothelial growth factor (VEGF) is a critical regulator (Ferrara, Gerber, \& LeCouter, 2003). VEGF acts as an angiogenic signal, as it increases both the number of blood vessels and their permeability, allowing oxygen to be transported effectively (Ferrara, et al., 2003). Circulating VEGF levels were increased after lowload resistance exercise combined with BFR, but no such increase in VEGF levels was observed following low-load resistance exercise alone (Patterson, Leggate, Nimmo, \& Ferguson, 2013; Shimizu, et al., 2016). It is speculated that during resistance exercise with BFR, hypoxic conditions occurring in compressed skeletal muscle contribute to increases in VEGF (Downs, et al., 2014; Ji, Mac Gabhann, \& Popel, 2007; Manini \& Clark, 2009).

Despite these multiple positive effects of BFR resistance exercise, some populations such as load-contraindicated individuals find it difficult to engage in active resistance training or to maintain even regular physical activity. Therefore, strategies that can invoke muscle contraction both conveniently and effectively to help people against physical inactivity might be of great significance. For example, the whole-body vibration (WBV) training devices are specially designed machines that contract users' muscles passively. They also have been used as an alternative training modality for many years. Several studies demonstrated that long-term WBV training effects were similar to those observed after resistance exercise, which led to increases in both muscle mass and strength (Martínez-Pardo, Romero-Arenas, Martínez-Ruiz, Rubio-Arias, \& Alcaraz, 2014; Osawa, Oguma, \& Ishii, 2013). Regarding WBV and muscular endurance topics, research is less extensive than that on the strength topic, and there is limited research on the VEGF issue. Previous studies investigated the effect of WBV on the VEGF response as evaluated by exercise with superimposed WBV (Beijer, et al., 2013; Suhr, et al., 2007). Previous findings of these seemed contradictory, and differences existed between exercise modes, with an additive effect on the circulating VEGF response was found when WBV was applied to endurance exercise compared with endurance exercise alone (Suhr, et al., 2007). In contrast, the VEGF response decreased when WBV was applied during resistance exercise (Beijer, et al., 2013). In addition, these studies may emphasize their cooperative effects; no previous study has investigated the effect of isolated WBV exposure on the VEGF response.

Collectively, WBV may potentially increase VEGF levels, the oxygen availability biomarker, through mechanical stimuli. More specifically, some WBV studies led to a training response comparable with those during resistance exercise and that resistance exercise performed under BFR conditions can increase circulating VEGF levels. Given that oxygen availability is typically related to $\mathrm{HR}$ and oxygen saturation $\left(\mathrm{SpO}_{2}\right)$, and the values of these markers were also magnified by BFR resistance exercise (Neto, et al., 2016; Takano, et al., 2005), provided the rationale for this study. Therefore, the purpose of this study is to compare the acute effects of WBV with and without BFR on the VEGF, HR, and $\mathrm{SpO}_{2}$ responses. We hypothesized that WBV + BFR would increase circulating VEGF levels, $\mathrm{HR}$, and $\mathrm{SpO}_{2}$ to a greater extent than that by WBV alone.

\section{Methods}

\section{Subjects}

In total, 10 healthy males (age: $21.3 \pm 1.15$ years, body height: $176.52 \pm 4.81 \mathrm{~cm}$, body mass: $69.12 \pm$ $7.14 \mathrm{~kg}$ ) participated in this study. All individuals were determined to be physically inactive through screening with the International Physical Activity Questionnaire-Short Form (Gauthier, Lariviere, \& Young, 2009). No participants reported that they were smokers or alcohol and coffee drinkers. The Institutional Review Board of Kaohsiung Medical University Chung-Ho Memorial Hospital approved all methods and procedures employed in this study, and all participants provided written informed consent. The sample size was calculated according to a previous study, which compared resistance exercise with or without BFR on the VEGF response using a repeated measures design (Shimizu, et al., 2016). In that study, a mean difference of $19.3 \mathrm{pg} / \mathrm{mL}$ (standard deviation; SD 19.5 $\mathrm{pg} / \mathrm{mL}$ ) was reported in the VEGF between resistance exercise + BFR and resistance exercise alone. Based on this study, sample size calculations indicated that 10 participants would be required to show an expected difference in the VEGF of this magnitude, using a power of 0.9 and an alpha level of 0.05 . 


\section{Study design}

A week before the experiment, participants were familiarized with the experimental procedures and devices. Subsequently, they completed two exercise sessions, i.e., WBV + BFR (intervention: WBV plus BFR) or WBV (intervention: WBV alone), using a repeated measures randomized crossover design, with sessions separated by a 1-week interval. The acute WBV protocol was adapted from Bosco et al. (2000), who reported that hormones were successfully induced by the WBV exposure, suggesting the treatment could provide an adequate exercise stimulus for individuals. In the WBV + BFR session, BFR was achieved via the application of external pressure using inflatable cuffs over the proximal portion of the thigh muscles.

To measure exercise intensity during each exercise, participants' HR measurements were conducted to document and compare any significant changes between the exercise sessions. To measure whether WBV or WBV + BFR would cause systematic hypoxia, the participants' $\mathrm{SpO}_{2}$ were measured. In addition, fasting blood samples before and after the exercise were collected to assess VEGF responses. For the experiments, participants were instructed to refrain from consuming alcohol, caffeine, or nutritional supplements for $24 \mathrm{~h}$ before the exercise sessions and to avoid strenuous exercise for $48 \mathrm{~h}$ before the sessions. To minimize diurnal variance, all measurements were obtained at the same time in the morning of the experiment day (between 9:00 and 11:00 a.m.).

\section{Procedure}

The study lasted for one month and was performed during summer. On each assessment day, participants arrived at the laboratory and rested for 10-15 min. The lowest HR recorded during this period was considered as resting HR. Then, a registered nurse collected fasting blood samples from the participants who were in the sitting position as a baseline measurement. Subsequently, participants performed a low-intensity warm-up on a cycle ergometer for $5 \mathrm{~min}$ at a self-selected comfortable pace (pedal cadence $<50$ revolutions per minute against no resistance). All subjects were asked to perform the warm-up in the same manner (pace) as they had done in the first session. Participants then completed a given WBV exercise with or without BFR, and they were instructed to stand on the vibration platform in a static squat position at $100^{\circ}$ of knee flexion with their hands placed on the rigid lever arms of the exercise platform during the exercise. At the end of each set, the participants' $\mathrm{HR}$ and level of $\mathrm{SpO}_{2}$ was measured. Immediately post-exercise, as well as 15 and 30 min post-exercise, blood samples for subsequent analysis were collected again from each participant who had resumed the sitting position.

\section{WBV protocol}

The WBV protocol was adapted from Bosco et al. (2000). In the current study, the participants were exposed to WBV exercise on a commercially available platform (BH YT18, Taipei, Taiwan). The vibration frequency was set at $26 \mathrm{~Hz}$ (amplitude $=$ $4 \mathrm{~mm}$ ). The participants were exposed to a series of 10 sets of WBV, each with a 1-min duration and 1-min rest between sets, except for 2 min of rest allowed after the fifth treatment. The WBV was performed with the participants standing on the vibration platform in a static squat position at $100^{\circ}$ of knee flexion with their hands placed on the rigid lever arms. During the rest intervals, participants were instructed to stand on the vibration platform. During all of the vibration treatments, the participants were instructed to wear thin socks, but no shoes.

\section{BFR device and pressure applied}

In the WBV + BFR session, all the participants used a custom-made BFR device, in which two cuffs were applied (Shenwang, New Taipei, Taiwan) around the proximal portion of the thigh muscles. The device is similar to a handheld sphygmomanometer, which is composed of two inflatable cuffs (made of nylon; width $=9 \mathrm{~cm}$, length $=70 \mathrm{~cm}$ ), a hand bulb pump with a check valve, a pressure gauge, and rubber tubes. The two cuffs are connected to the hand bulb pump by the rubber tubes, through which air passes during pumping. Another tube from the bulb connects the pressure gauge, in which the pressure of the cuffs is obtained.

Although during the BFR resistance exercise the cuff pressure is considered to be adjusted based on the individual's limb circumference, recent evidence has shown that limb circumference has a limited impact on the cuff pressure required for partial BFR (Hunt, Stodart, \& Ferguson, 2016). The appropriate cuff pressure to be combined with WBV for a beginner to perform the WBV + BFR exercise is not well known. A uniform cuff pressure of $200 \mathrm{mmHg}$ has been used for recreationally active men performing WBV exercise in a static half-squat position (Aguayo, et al., 2016). However, the pressure may not be suitable for beginners because previous studies on frail individuals (elderly and untrained subjects) have not utilized a cuff pressure of $200 \mathrm{mmHg}$ for lowerlimb exercise during the early phase of training (Abe, et al., 2010; Ozaki, Miyachi, Nakajima, \& Abe, 2011a; Ozaki, et al., 2011b). Ozaki et al. (2011a, 2011b) applied a cuff pressure that ranged from 140 to $200 \mathrm{mmHg}$ on untrained elderly individuals in 10 weeks of BFR training session. In these studies, on the first day of training, the cuff pressure was set at $140 \mathrm{mmHg}$. The cuff pressure setting at 140 $\mathrm{mmHg}$ on the first day of training was also seen in Abe et al. (2010) who used active elderly individ- 
uals as participants. In addition, a previous study has recommended that a cuff pressure of 140-160 $\mathrm{mmHg}$ is considered suitable for most individuals (Fahs, Loenneke, Rossow, Thiebaud, \& Bemben, 2012). Hence, in the pilot work by our study group, cuffs were inflated to a pressure of $140-160 \mathrm{mmHg}$ with participants standing (Pstand). Then, participants postured themselves in a static squat position with $100^{\circ}$ of knee flexion with their hands placed on the rigid lever arms of the vibration device. From a standing to a squatting position, the thigh circumference increases, which will tighten the BFR application, consequently resulting in elevating the cuff pressure (Psquat). After testing the protocol several times, we concluded that participants would not tolerate 10 sets of WBV + BFR once the cuff pressure was above approximately $190-200 \mathrm{mmHg}$ in the Psquat, which was initially set at $150 \mathrm{mmHg}$ in the Pstand. For both the safety reasons and to ensure that all participants completed the protocol, the cuff pressure of Pstand utilized in the current study was set at $140 \mathrm{mmHg}$. During the WBV + BFR session, the cuff pressure of Psquat was elevated to $180-190$ $\mathrm{mmHg}$. Once cuffs were inflated, they remained so for the entire experimental session, including rest intervals between the sets. Also, pressure of the cuff was adjusted manually by the supervisor to fit each participant's Pstand or Psquat within the range of $\pm 3 \mathrm{mmHg}$.

\section{HR and level of oxygen saturation}

The participants' HR and level of $\mathrm{SpO}_{2}$ were measured before and during the exercise using a finger oximeter (MD-680P Comdek, Taiwan). Immediately at the end of each set of exercise sessions, $\mathrm{HR}$ and $\mathrm{SpO}_{2}$ values were recorded and averaged for statistical analysis. HR values were further expressed as age-adjusted (220 minus age) maximal heart rate percentage (\% HRmax) to represent relative exercise intensity for each trial.

\section{Blood sample collection and analysis}

Blood samples were collected from the antecubital vein, both before and after each exercise session. Blood samples were stored at $4^{\circ} \mathrm{C}$ and centrifuged at $1500 \mathrm{rpm}$ for $30 \mathrm{~min}$ within $2 \mathrm{~h}$ of sampling. Subsequently, the serum samples were stored at $2^{\circ} \mathrm{C}$ for VEGF assays. VEGF levels were analyzed using human VEGF quantikine enzymelinked immunosorbent assay kit (R\&D Systems, Inc., Minneapolis, MN, USA). The sensitivity, as well as both interassay and intra-assay coefficients of variance, were $5.0 \mathrm{pg} / \mathrm{mL}, 4.5-6.7 \%$, and $6.2-8.8 \%$, respectively.

\section{Statistical analyses}

Data are expressed as the mean \pm standard error (SE). All dependent variables were analyzed using a two-way analysis of variance with repeated measures (trial vs. time). Post-hoc analysis was performed using the least significant difference (LSD) technique. Statistical significance was set at $\mathrm{p}<.05$ for all tests. All statistical analyses were conducted using SPSS software program 22.0 (SPSS Inc. Chicago, USA).

\section{Results}

\section{Heart rate}

Acute changes in HR and age-adjusted \% HRmax values are shown in Table 1. The significant difference was determined between time ( $\mathrm{F}=19.26, \mathrm{p}=.002$, effect size, $\mathrm{ES}=0.68$ for HR; $\mathrm{F}=20.15, \mathrm{p}=.002, \mathrm{ES}=0.69$ for $\%$ HRmax); time $\times$ trial interaction $(\mathrm{F}=32.07, \mathrm{p}<.001, \mathrm{ES}=0.78$ for HR; $\mathrm{F}=29.40, \mathrm{p}<.001, \mathrm{ES}=0.77$ for $\% \mathrm{HRmax}$ ); and treatment $(\mathrm{F}=956.04, \mathrm{p}<.001, \mathrm{ES}=0.99$ for HR; $\mathrm{F}=839.33, \mathrm{p}<.001, \mathrm{ES}=0.99$ for $\% \mathrm{HRmax}$ ) at HR and \% HRmax values. Post-hoc comparisons revealed that pre-exercise values of $\mathrm{HR}$ and $\%$ HRmax did not differ significantly between WBV and $\mathrm{WBV}+\mathrm{BFR}$ sessions $(\mathrm{p}=.78, \mathrm{ES}=0.03$ for $\mathrm{HR}$; $\mathrm{p}=.78, \mathrm{ES}=0.03$ for $\%$ HRmax). During the exercise, HR and \% HRmax increased significantly from pre-exercise levels for both the WBV + BFR $(p<.001, E S=7.4$ for HR and 7.27 for \% HRmax) and WBV ( $p<.001, E S=6.69$ for HR and 6.77 for $\%$ HRmax) sessions. Also, during the exercise, both $\mathrm{HR}$ and \% HRmax values were higher for the WBV + BFR sessions than for the WBV sessions ( $\mathrm{p}=.001$, $\mathrm{ES}=1.57$ for $\mathrm{HR}$ and $\mathrm{ES}=1.56$ for \% HRmax).

\section{Oxygen saturation}

The $\mathrm{SpO}_{2}$ data are shown in Table 1. No significant time $(\mathrm{F}=0.125, \mathrm{p}=.73, \mathrm{ES}=0.01), \operatorname{trial}(\mathrm{F}=0.045$, $\mathrm{p}=.837, \mathrm{ES}<0.01$ ), or time $\times$ trial interaction $(\mathrm{F}=1.359, \mathrm{p}=.274, \mathrm{ES}=0.13)$ effects were observed for $\mathrm{SpO}_{2}$.

\section{VEGF}

While the two-way ANOVA with repeated measures showed that VEGF values indicated a significant main effect for time $(\mathrm{F}=6.26, \mathrm{p}=.002$, $\mathrm{ES}=0.41)$ and a time $\times$ trial interaction $(\mathrm{F}=4.07$, $\mathrm{p}=.017, \mathrm{ES}=0.31$ ), no evidence was found for trial effect $(\mathrm{F}=0.56, \mathrm{p}=.467, \mathrm{ES}=0.04)$. An LSD posthoc comparison among time points revealed that compared with the baseline measurements, the VEGF value was significantly higher immediately post-exercise $(p=.020, E S=0.43)$ only in the WBV + BFR session, and returned to the pre-exercise value at $15 \mathrm{~min}(\mathrm{p}=.71, \mathrm{ES}<0.01)$ post-exercise. In addition, an LSD post-hoc comparison between trials revealed that while the pre-exercise value for VEGF did not differ between the sessions ( $\mathrm{p}=.782$, $\mathrm{ES}=0.04$ ), the VEGF values for $\mathrm{WBV}+\mathrm{BFR}$ were 
Table 1. Physiological variables between $W B V+B F R$ and $W B V$

\begin{tabular}{llccccc}
\hline & Trial & Pre-exercise & During exercise & Time & Time $\times$ & Trial \\
\hline HR & WBV+BFR & $71.70 \pm 1.17$ & $113.71 \pm 2.25 \# \dagger$ & $19.26^{*}$ & $32.07^{*}$ & $956.04^{*}$ \\
$\%$ beat/min) & WBV & $71.60 \pm 0.93$ & $103.37 \pm 1.91$ & & & \\
& WBV+BFR & $36.08 \pm 0.59$ & $57.23 \pm 1.15 \# \dagger$ & $20.15^{*}$ & $29.40^{*}$ & $839.33^{*}$ \\
& WBV & $36.04 \pm 0.46$ & $52.02 \pm 0.94$ & & & \\
$\mathrm{SpO}_{2}(\%)$ & WBV+BFR & $98.60 \pm 0.16$ & $98.48 \pm 0.16$ & 0.12 & 1.32 & 0.04 \\
& WBV & $98.40 \pm 0.16$ & $98.57 \pm 0.12$ & & & \\
\hline
\end{tabular}

Note. ${ }^{*} p<.05$, compared between WBV+BFR and WBV (two-way repeated measures ANOVA). \#p<.05, significantly different from pretraining levels. $\uparrow p<.05$, significantly different from the WBV during exercise. $W B V+B F R=$ whole body vibration plus blood flow restriction; WBV = whole body vibration; $\mathrm{HR}=$ heart rate.

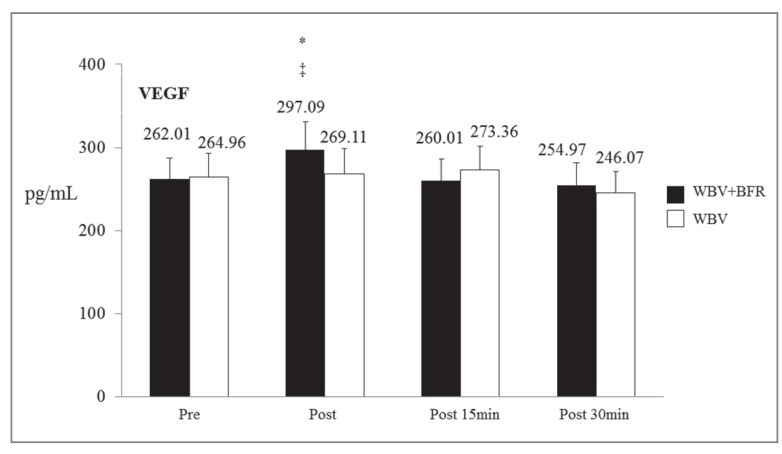

Figure 1. Comparison of blood VEGF concentrations (mean $\pm S E$ ) between $W B V+B F R$ and $W B V$ for various time points. * significant difference between $W B V+B F R$ and $W B V ;$ significant difference compared with baseline measurements; $W B V+B F R=$ whole body vibration plus blood flow restriction; $W B V=$ whole body vibration.

significantly higher $(p=.02, E S=0.28)$ at the time point immediately post-exercise than those for WBV (Figure 1).

\section{Discussion and conclusions}

The current study demonstrated that in untrained men, WBV + BFR induced a greater HR response than WBV exercise alone. However, WBV exercise with or without BFR failed to cause significant changes in $\mathrm{SpO}_{2}$. In addition, circulating VEGF increased post-exercise only in the WBV + BFR session, suggesting cooperative effects, whereas no increase was observed in WBV.

In the present study, we applied BFR to WBV, as an alternative to increasing workload, because previous studies indicated that strategies aiming to increase the desired effects of WBV training effects often employ external resistance, which can magnify the acute WBV exercise response (Giunta, et al., 2013; Pojskic, et al., 2015). In the present study, the participants' HRs was assessed to evaluate the exercise intensity between the two exercise sessions. The results revealed that when applying BFR to WBV, HR increased to a greater extent (from $52.02 \%$ to $57.23 \% \mathrm{HRmax}$ ). The results of this study are similar to those of previous studies that used external BFR during resistance exercise (Neto, et al., 2016; Takano, et al., 2005). It is speculated that during exercise with BFR, the restriction to venous return and/or increased vascular resistance was manifested in a reduced stroke volume and a simultaneous increase in HR to maintain cardiac output (Renzi, Tanaka, \& Sugawara, 2010; Takano, et al., 2005).

Systemic oxygen deficiency may facilitate endurance-type exercise-induced adaptation (Montero \& Lundby, 2016). In our current study, although WBV + BFR elicited a significantly greater HR response versus the non-BFR WBV condition, neither WBV nor WBV + BFR sessions caused significant changes in $\mathrm{SpO}_{2}$. As Nielsen (2003) has indicated, exercise intensity determines the degree of hypoxemia. Likewise, Seixas, Pereira, Moreira, and Paschoal (2013) demonstrated that oxygen desaturation was observed after intense physical activity (more than $85 \%$ HRmax). Currently, we can only speculate that the exercise intensity of the two regimens applied in our study did not elicit enough stimuli, as the relative exercise intensity for the WBV + BFR was only $57.23 \%$ HRmax. The intensity may not cause systematic hypoxia since evidence has indicated that individuals who did not exercise at greater than $73 \% \mathrm{VO}_{2}$ max (approximately $85 \%$ HRmax) during 60 min of endurance exercise failed to display hypoxemia (Stewart \& Pickering, 2007). In addition, the applied exercise mode should also be considered because the amount of muscle mass involved in the exercise may influence oxygen desaturation. Neto et al. (2016) employed a BFR combined with upper- and lowerlimb resistance exercise regimens (consisting of biceps curls, triceps forehead extensions, and both knee extension and flexion) to be performed several repetitions (30 repetitions followed by three sets of 15 repetitions) and separated by a short rest interval ( $30 \mathrm{~s}$ of rest between all sets and $1 \mathrm{~min}$ of rest between exercises). In contrast to our study, Neto et al. (2016) found that $\mathrm{SpO}_{2}$ decreased following acute, low-load resistance exercise combined with BFR. In our study, we used WBV exercise, which 
only emphasized the lower limbs, particularly the quadriceps. The different mode applied may have contributed to the observed discrepancies between the studies.

It is well established that VEGF is a critical regulator in promoting angiogenesis, serving as an indirect measurement of capillarization (Ferrara, et al., 2003; Prior, Yang, \& Terjung, 2004). The VEGF response to WBV has been examined by WBV superimposed on different types of exercise, but the results have been inconsistent according to the mode applied. For example, elevations were found in endurance exercise (Suhr, et al., 2007), but reductions were observed in resistance exercise (Beijer, et al., 2013). In the current study, in the session of WBV exercise alone, only the WBV treatment was applied. The results indicated that there was no change in VEGF level upon WBV exposure, suggesting that WBV alone did not cause a significant change in VEGF levels. In addition, the increase in VEGF following WBV + BFR would, therefore, support recent findings of previous occlusion training studies (resistance exercise combined with BFR mode) that have observed a magnified increase in the circulating VEGF level (Shimizu, et al., 2016).

Two primary regulators have been proposed as causal factors in the exercise-induced VEGF response, including mechanical forces, which mostly refer to shear stress during muscle activity, and oxygen availability (Hoier \& Hellsten, 2014). Vibration itself is considered to produce shear stress (Suhr, et al., 2007; Yue \& Mester, 2007), which may be a potent stimulator of VEGF. However, our data revealed that WBV exercise alone did not induce an increase in VEGF levels, suggesting that the vibration-induced shear stress in the current study was unable to stimulate a VEGF response. Regarding BFR exercise, BFR + resistance exercise has been reported to cause less shear stress due to low blood flow caused by cuff compression compared with that of non-BFR resistance exercise (Takano, et al., 2005). Therefore, it could be speculated that the increased VEGF levels, caused by adding the application of BFR to WBV exercise in the current study, may predominantly rely on oxygen availability rather than shear stress.

Studies on the link between oxygen availability and exercise-induced VEGF have been attributed to hypoxic episodes (Semenza, 1999). However, neither the WBV nor the WBV + BFR session in our study caused a significant change in $\mathrm{SpO}_{2}$, suggesting that neither exercise mode led to a systemic oxygen deficit. Despite this, previous studies have suggested that during resistance exercise with BFR, local muscle oxygen tension may fall considerably due to an insufficient oxygen supply from low blood flow, followed by the secretion of VEGF immediately after a single bout of exercise (Ji, et al., 2007; Manini \& Clark, 2009). Consistent with these viewpoints, Downs et al. (2014) indicated that the hypoxic environment accelerates VEGF release from cells in the vascular endothelium and skeletal muscles. Taken together, these lines of evidence suggest that hypoxic stress in working muscles may occupy the primary role of VEGF secretion induced by WBV + BFR.

Regarding the results obtained in the present study, some limiting factors become relevant and warrant explanation. First, although we measured $\mathrm{SpO}_{2}$, we did not measure skeletal muscle oxygen tension, which, if measured, would provide further insight into the mechanisms behind these responses. Second, although the cuff pressure was adjusted based on an individual's limb circumference as recommended by Fahs et al. (2012), the majority of previous studies on frail individuals (elderly and untrained subjects) and BFR exercise novices have often utilized a cuff pressure of 140 $\mathrm{mmHg}$ for lower-limb exercise (Abe, et al., 2010; Ozaki, et al., 2011a, 2011b). For safety reasons, the uniform cuff pressure of $140 \mathrm{mmHg}$ was applied to all participants. In the present study, although all participants were physically inactive, a uniform pressure was applied to each participant, and the WBV-magnified exercise response was observed so that individual differences in exercise tolerance were observed. This might lead to a disproportionate increase in blood parameter values. Last, defying definite conclusions about the benefits of a short-term training program on muscular endurance performance is beyond the scope of the research design; we are only left to speculate that WBV + BFR following a period of training might have the potential effects to improve muscular endurance among people who engage in low levels of physical activity.

In conclusion, an acute bout of WBV exercise failed to cause significant changes in circulating VEGF concentrations. However, WBV exercise by superimposition of external pressure using inflatable cuffs proximal to the working muscle enhanced the HR response and induced increases in circulating VEGF levels. These alterations seem unrelated to systematic hypoxia, as $\mathrm{SpO}_{2}$ did not change with either the WBV or WBV + BFR intervention. In addition, because increased VEGF levels can facilitate effective oxygen transport in skeletal muscle, i.e., if untrained participants want to enhance the WBV exercise-induced muscular endurance response, they can compress the proximal parts of the working muscles with the appropriate pressure. 


\section{References}

Abe, T., Sakamaki, M., Fujita, S., Ozaki, H., Sugaya, M., Sato, Y., \& Nakajima, T. (2010). Effects of low-intensity walk training with restricted leg blood flow on muscle strength and aerobic capacity in older adults. Journal of Geriatric Physical Therapy, 33(1), 34-40.

Aguayo, D., Mueller, S.M., Boutellier, U., Auer, M., Jung, H.H., Flück, M., \& Toigo, M. (2016). One bout of vibration exercise with vascular occlusion activates satellite cells. Experimental Physiology, 101(2), 295-307.

Beijer, A., Rosenberger, A., Bölck, B., Suhr, F., Rittweger, J., \& Bloch, W. (2013). Whole-body vibrations do not elevate the angiogenic stimulus when applied during resistance exercise. PLosOne, 8(11), e80143.

Bosco, C., Iacovelli, M., Tsarpela, O., Cardinale, M., Bonifazi, M., Tihanyi, J., Viru, M., De Lorenzo, A., \& Viru, A. (2000). Hormonal responses to whole-body vibration in men. European Journal of Applied Physiology, 281(6), 449-454.

Downs, M.E., Hackney, K.J., Martin, D., Caine, T.L., Cunningham, D., O’Connor, D.P., \& Ploutz-Snyder, L.L. (2014). Acute vascular and cardiovascular responses to blood flow-restricted exercise. Medicine and Science in Sports and Exercise, 46(8), 1489-1497.

Evans, C., Vance, S., \& Brown, M. (2010). Short-term resistance training with blood flow restriction enhances microvascular filtration capacity of human calf muscles. Journal of Sports Sciences, 28(9), 999-1007.

Fahs, C.A., Loenneke, J.P., Rossow, L.M., Thiebaud, R.S., \& Bemben, M.G. (2012). Methodological considerations for blood flow restricted resistance exercise. Journal of Trainology, 1(3), 14-22.

Ferrara, N., Gerber, H.P., \& LeCouter, J. (2003). The biology of VEGF and its receptors. Nature Medicine, 9(6), 669-676.

Gauthier, A.P., Lariviere, M., \& Young, N. (2009). Psychometric properties of the IPAQ: A validation study in a sample of northern Franco-Ontarians. Journal of Physical Activity and Health, 6(Suppl. 1), S54-S60.

Giunta, M., Rigamonti, A.E., Agosti, F., Patrizi, A., Compri, E., Cardinale, M., \& Sartorio, A. (2013). Combination of external load and whole body vibration potentiates the GH-releasing effect of squatting in healthy females. Hormone and Metabolic Research, 45(8), 611-616.

Hoier, B., \& Hellsten, Y. (2014). Exercise-induced capillary growth in human skeletal muscle and the dynamics of VEGF. Microcirculation, 21(4), 301-314.

Hunt, J.E., Stodart, C., \& Ferguson, R.A. (2016). The influence of participant characteristics on the relationship between cuff pressure and level of blood flow restriction. EuropeanJournal of Applied Physiology, 116(7), 1421-1432.

Ji, J.W., Mac, Gabhann.F., \& Popel, A.S. (2007). Skeletal muscle VEGF gradients in peripheral arterial disease: Simulations of rest and exercise. American Journal of Physiology, 293(6), H3740-H3749.

Kacin, A., \& Strazer, K. (2011). Frequent low-load ischemic resistance exercise to failure enhances muscle oxygen delivery and endurance capacity. Scandinavian Journal of Medicine and Science in Sports, 21(6), 231-241.

Kim, D., Loenneke, J.P., Ye, X., Bemben, D.A., Beck, T.W., Larson, R.D., \& Bemben, M.G. (2017). Low-load resistance training with low relative pressure produces muscular changes similar to high-load resistance training. Muscle Nerve,56(6), E126-E133.

Loenneke, J.P., \& Pujol, T.J. (2009). The use of occlusion training to produce muscle hypertrophy. Strength and Conditioning Journal, 31(3), 77-84.

Loenneke, J.P., Young, K.C., Wilson, J.M., \& Andersen, J.C. (2013). Rehabilitation of an osteochondral fracture using blood flow restricted exercise: A case review. Journal of Bodywork and Movement Therapies, 17(1), 42-45.

Manini, T.M., \& Clark, B.C. (2009). Blood flow restricted exercise and skeletal muscle health. Exercise and Sport Sciences Reviews, 37(2), 78-85.

Martínez-Pardo, E., Romero-Arenas, S., Martínez-Ruiz, E., Rubio-Arias, J.A., \& Alcaraz, P.E. (2014). Effect of a whole-body vibration training modifying the training frequency of workouts per week in active adults. Journal of Strength and Conditioning Research, 28(11), 3255-3263.

Montero, D., \& Lundby, C. (2016). Effects of exercise training in hypoxia versus normoxia on vascular health. Sports Medicine, 46(11), 1725-1736.

Neto, G.R., Sousa, M.S., Costa e Silva, G.V., Gil, A.L., Salles, B.F., \& Novaes, J.S. (2016). Acute resistance exercise with blood flow restriction effects on heart rate, double product, oxygen saturation and perceived exertion. Clinical Physiology and Functional Imaging, 36(1), 53-59.

Nielsen, H.B. (2003). Arterial desaturation during exercise in man: Implication for O2 uptake and work capacity. Scandinavian Journal of Medicine and Science in Sports, 13(6), 339-358.

Osawa, Y., Oguma, Y., \& Ishii, N. (2013). The effects of whole-body vibration on muscle strength and power: A metaanalysis. Journal of Musculoskeletal and Neuronal Interactions, 13(3), 380-390.

Ozaki, H., Miyachi, M., Nakajima, T., \& Abe, T. (2011a). Effects of 10 weeks walk training"with leg blood flow reduction on carotid arterial compliance and muscle size in the elderly adults. Angiology, 62(1), 81-86.

Ozaki, H., Sakamaki, M., Yasuda, T., Fujita, S., Ogasawara, R., Sugaya, M., Nakajima, T., \& Abe, T. (2011b). Increases in thigh muscle volume and strength by walk training with leg blood flow reduction in older participants. The Journals of Gerontology. Series A, Biological Sciences and Medical Sciences, 66(3), 257-263. 
Patterson, S.D., Leggate, M., Nimmo, M.A., \& Ferguson, R.A. (2013). Circulating hormone and cytokine response to low-load resistance training with blood flow restriction in older men. European Journal of Applied Physiology, 113(3), 713-719.

Pojskic, H., Pagaduan, J., Uzicanin, E., Babajic, F., Muratovic, M., \& Tomljanovic, M. (2015). Acute effects of loaded whole body vibration training on performance. Asian Journal of Sports Medicine, 6(1), e24054.

Pope, Z.K., Willardson, J.M., \& Schoenfeld, B.J. (2013). Exercise and blood flow restriction. Journal of Strength and Conditioning Research, 27(10), 2914-2926.

Prior, B.M., Yang, H.T., \& Terjung, R.L. (2004). What makes vessels grow with exercise training? Journal of Applied Physiology, 97(3), 1119-1128.

Renzi, C.P., Tanaka, H., \& Sugawara, J. (2010). Effects of leg blood flow restriction during walking on cardiovascular function. Medicine and Science in Sports and Exercise, 42(4), 726-732.

Scott, B.R., Loenneke, J.P., Slattery, K.M., \& Dascombe, B.J. (2016). Blood flow restricted exercise for athletes: A review of available evidence. Journal of Science and Medicine in Sport, 19(5), 360-367.

Seixas, D.M., Pereira, M.C., Moreira, M.M., \& Paschoal, I.A. (2013). Oxygen desaturation in healthy subjects undergoing the incremental shuttle walk test. Jornal Brasileiro de Pneumologia, 39(4), 440-446.

Semenza, G.L. (1999). Regulation of mammalian O2 homeostasis by hypoxia-inducible factor 1. Annual Review of Cell and Developmental Biology, 15, 551-578.

Shimizu, R., Hotta, K., Yamamoto, S., Matsumoto, T., Kamiya, K., Kato, M., Hamazaki, N., Kamekawa, D., Akiyama, A., Kamada, Y., Tanaka, S., \& Masuda T. (2016). Low -intensity resistance training with blood flow restriction improves vascular endothelial function and peripheral blood circulation in healthy elderly people. European Journal of Applied Physiology, 116(4), 749-757.

Stewart, I.B., \& Pickering, R.L. (2007). Effect of prolonged exercise on arterial oxygen saturation in athletes susceptible to exercise-induced hypoxemia. Scandinavian Journal of Medicine \& Science in Sports, 17(4), 445-451

Suhr, F., Brixius, K., de Marées, M., Bölck, B., Kleinöder, H., Achtzehn, S., Bloch, W., \& Mester, J. (2007). Effects of short-term vibration and hypoxia during high-intensity cycling exercise on circulating levels of angiogenic regulators in humans. Journal of Applied Physiology, 103(2), 474-483.

Takano, H., Morita, T., Iida, H., Asada, K., Kato, M., Uno, K., Hirose, K., Matsumoto, A., Takenaka, K., Hirata, Y., Eto, F., Nagai, R., Sato, Y., \& Nakajima, T. (2005). Hemodynamic and hormonal response to a short-term low-intensity resistance exercise with a reduction in muscle blood flow. European Journal of Applied Physiology, 95(1), 65-73.

Takarada, Y., Takazawa, H., \& Ishii, N. (2000a). Application of vascular occlusion diminish disuse atrophy of knee extensor muscles. Medicine and Science in Sports and Exercise, 32(12), 2035-2039.

Takarada, Y., Takazawa, H., Sato, Y., Takebayashi, S., Tanaka, Y., \& Ishii, N. (2000b). Effects of resistance exercise combined with moderate vascular occlusion on muscle function in humans. Journal of Applied Physiology, 88(6), 2097-2106.

Vechin, F.C., Libardi, C.A., Conceição, M.S., Damas, F.R., Lixandrão, M.E., Berton, R.P., Tricoli, V.A., Roschel, H.A., Cavaglieri, C.R., Chacon-Mikahil, M.P., \& Ugrinowitsch, C. (2015). Comparisons between low-intensity resistance training with blood flow restriction and high-intensity resistance training on quadriceps muscle mass and strength in elderly. Journal of Strength and Conditioning Research, 29(4), 1071-1076.

Yue, Z., \& Mester, J. (2007). On the cardiovascular effects of whole-body vibration part I. Longitudinal effects: Hydrodynamic analysis. Studies in Applied Mathematics, 119(2), 95-109.

Submitted: December 6, 2016

Accepted: February 19, 2018

Published Online First: August 31, 2018

Correspondence to:

Assist. Prof. Cai Zong-Yan, Ph.D.

Division of Physical and Health Education

Center for General Education

National Sun Yat-sen University

No.70, Lianhai Rd., Gushan Dist.

Kaohsiung City 804, Taiwan

Fax: +886-3-5255-851

Phone: +886-7-5252-000, ext 5872

E-mail: flameyan@yahoo.com.tw

\section{Acknowledgments}

No financial assistance or grant support was obtained for this study. The authors appreciate the contribution of all participants. 